GP39

A CASE SERIES OF SUSPECTED CONGENITAL ADRENAL HYPERPLASIAIN ONE WEEK IN A REGIONAL ENDOCRINE UNIT

${ }^{1}$ Pankaj Agrawal*, ${ }^{1}$ Aisling McCann, ${ }^{1}$ Kevin Conlon, ${ }^{1}$ Claire Reynolds, ${ }^{1}$ Caroline Joyce, ${ }^{2}$ Norman Taylor, ${ }^{1}$ Stephen MP O'Riordan. ${ }^{1}$ Cork University Hospital, Cork, Ireland; ${ }^{2}$ Kings College Hospital, London, UK

\subsection{6/archdischild-2019-epa.105}

Congenital adrenal hyperplasia (CAH) is the most common cause of salt wasting during infancy. During workup other rarer causes should also be considered. We report three cases of suspected $\mathrm{CAH}$, aiming to highlight that $\mathrm{CAH}$ is not always the cause of adrenal insufficiency.

Case $1 \mathrm{~A}$ two-week-old term female born to non-consanguineous parents, presented with failure to thrive, hyponatremia, $\mathrm{Na} 118 \mathrm{mmol} / \mathrm{L}$ (132-144 mmol/L), hyperkalaemia, K $7.2 \mathrm{mmol} / \mathrm{L}(3.5-5.1 \mathrm{mmol} / \mathrm{L})$, plasma (am) cortisol 134 $\mathrm{nmol} / \mathrm{L}(101-536 \mathrm{nmol} / \mathrm{L})$. A diagnosis of CAH was considered and Hydrocortisone (HC), Fludrocortisone and Sodium Chloride $(\mathrm{NaCl}) \quad(30 \%)$ were commenced. Urinary steroid profile (USP) was sent to Kings College, London diagnosing Aldosterone Synthase Deficiency (ASD) type I, prompting to stop HC and to continue Fludrocortisone. Further testing showed high renin $>300 \mathrm{pg} / \mathrm{ml} /$ hour (normal $<61 \mathrm{pg} / \mathrm{ml} /$ hour), low aldosterone $50 \mathrm{pg} / \mathrm{ml}$ (normal $>300-1900 \mathrm{pg} /$ $\mathrm{ml}$ ). 17-OH Progesterone and Adrenocorticotropic-hormone (ACTH) were normal.

Case 2 A term $3.7 \mathrm{~kg}$ female, antenatally diagnosed bilateral cleft lip \& palate, delivered by cesarean-section, to 44-yearold primi mother was admitted to NICU on day-3 with low Sodium $122 \mathrm{mmol} / \mathrm{L}(132-144 \mathrm{mmol} / \mathrm{L})$, high Potassium 6.2 $\mathrm{mmol} / \mathrm{L}(3.5-5.1 \mathrm{mmol} / \mathrm{L})$ normal Cortisol (am) 377nmol/L (101-536nmol/L), serum/urine osmolality. CAH was suspected and $\mathrm{HC}$, Fludrocortisone and $\mathrm{NaCl}$ (30\%) were commenced. MRI brain showed partial agenesis of corpus callosum, with normal pituitary. Further investigations: am cortisol: $85 \mathrm{nmol} / \mathrm{L}$ (101-536nmol/L), ACTH (am): 23.1 $\mathrm{pmol} / \mathrm{L}$ (1.1-13.2pmol/L), aldosterone, renin, $17 \mathrm{OH}$ progesterone, USP, urine-biochemistry, LH, FSH, B-HCG, AMH, TFT's \& Blood glucose were normal. MicroarrayCGH showed 16p13.11 microdeletion (Father same deletion), karyotype 46XX. Trial of stopping Fludrocortisone \& $\mathrm{NaCl}(30 \%)$ was unsuccessful and $\mathrm{NaCl}(30 \%)$ was recommenced. She failed a synachten test and remains on $\mathrm{HC}$ and $\mathrm{NaCl}(30 \%)$. Current diagnosis is adrenal insufficiency related to $16 \mathrm{p} 13.11$ microdeletion.

Case 3 A term 23 day old male was transferred to Cork University Hospital (CUH) from a peripheral hospital with 12 hours of projectile, non-bilious vomiting. On examination he was dehydrated, unwell, with hyper-pigmented scrotum (normal descended testes) and normal phallus. Initial tests revealed hyponatremia, $\mathrm{Na} 108 \mathrm{mmol} / \mathrm{L} \quad(132-144$ $\mathrm{mmol} / \mathrm{L})$, \& hyperkalemia, K $6.9 \mathrm{mmol} / \mathrm{L}$ (3.5-5.1 mmol/L). $\mathrm{CAH}$ was suspected and $\mathrm{HC}\left(8-10 \mathrm{mg} / \mathrm{m}^{2} /\right.$ day $)$, Fludrocortisone $200 \mathrm{mcg} \& \mathrm{NaCl}(30 \%)$ were commenced. $17-\mathrm{OH}$ progesterone was $>400 \mathrm{nmol} / \mathrm{L} \quad(<4 \mathrm{nmol} / \mathrm{L})$. Genetic tests confirmed a diagnosis of $\mathrm{CAH}$ secondary to 21-hydroxylase deficiency.

Conclusion All three cases presented within a week in CUH with acute adrenal insufficiency with salt wasting, thought to be CAH. However; only one was confirmed CAH. During the workup, USP and genetic testing were key to distinguish these rarer diagnoses.

\section{GP40 OHVIRA SYNDROME- AN UNUSUAL VARIANT IN AN INFANT}

Nikita Deegan*, Geraldine Connolly. Temple street Childrens University Hospital, Dublin Ireland

10.1136/archdischild-2019-epa.106

Obstructed hemivagina and ipsilateral renal anomaly (OHVIRA) syndrome is a rare Mullerian duct anomaly. The classical description is that of a uterus didelphys, unilateral obstructed hemivagina caused by a longitudinal sagittal vaginal septum and ipsilateral renal agenesis. An updated classification allows for inclusion of a wide variety of renal anomalies such as dysplastic kidneys, pelvic kidneys, or ectopic ureters.

We report a rare case of OHVIRA syndrome in a fourteen month old girl, with a single normal uterus, obstructed hemivagina with a longitudinal coronal vaginal septum and unilateral dysplastic kidney. Antenatal ultrasound scan at twenty weeks of gestation revealed a suspected hydrocolpos and dysplastic kidney. The infant was born at term via elective caesarean section (maternal request for elective repeat caesarean). Of note mum had a personal history of uterus didelphys which was diagnosed at her first delivery (emergency caesarean section). Mum had no known associated renal anomaly.

The patient was reviewed at five weeks old in the paediatric and adolescent gynaecology (PAG) clinic. She was thriving, but had a small vaginal bulge on examination. Repeat abdominal ultrasound scan revealed a single uterus, a normal right kidney and dysplastic left kidney, and a small hydrocolpos. As the patient was asymptomatic this was managed conservatively, and mum was advised to contact the PAG service if any concerns. At twelve months of age mum reported copious grey non-odorous discharge into the patient's nappy. This was thought to be the hydrocolpos draining spontaneously and the patient was called in for review in the PAG clinic, however on clinical examination a large mass was palpable to the umbilicus. Repeat ultrasound suggested a persistent large hydrocolpos $(10 \times 6 \times 4 \mathrm{~cm})$.

Examination under anaesthesia was performed. This demonstrated an elongated vagina with no cervix palpable at the superior aspect. A bulging septum in the coronal plane was then noted and a small punctum identified just inferior to the urethral orifice. This was opened draining $300 \mathrm{mls}$ of fluid. A single cervix was located in this obstructed anterior hemivagina.

Clinical and sonographic review at six weeks post-operative confirmed a single uterus, unilateral dysplastic kidney and no recurrence of hydrocolpos or discharge and the infant was thriving.

Cases of OHVIRA syndrome with variations in the classically described anatomical anomalies found in OHVIRA have been reported, however our patients variant with a single normal uterus, and a coronal septum may be a new variant not previously identified. 